\title{
Investigating Ghanaian Allium Species for Anti-Infective and Resistance-Reversal Natural Product Leads to Mitigate Multidrug-Resistance in Tuberculosis
}

\author{
Cynthia Amaning Danquah ${ }^{1, * \mathbb{D}}$, Michael Tetteh ${ }^{1}$, Isaac Kingsley Amponsah ${ }^{2}$, Abraham Yeboah Mensah ${ }^{2}$, \\ Kwame Ohene Buabeng ${ }^{3 \oplus}$, Simon Gibbons ${ }^{4}$ and Sanjib Bhakta ${ }^{5}[$ \\ 1 Department of Pharmacology, Faculty of Pharmacy and Pharmaceutical Sciences, Kwame Nkrumah \\ University of Science and Technology (KNUST), PMB, Kumasi, Ghana; mitetteh26@gmail.com \\ 2 Department of Pharmacognosy, Faculty of Pharmacy and Pharmaceutical Sciences, KNUST, PMB, \\ Kumasi, Ghana; akila.amponsah@gmail.com (I.K.A.); aymensah.pharm@knust.edu.gh (A.Y.M.) \\ 3 Department of Pharmacy Practice, Faculty of Pharmacy and Pharmaceutical Sciences, KNUST, PMB, \\ Kumasi, Ghana; kobuabeng.pharm@knust.edu.gh \\ 4 Norwich Research Park, School of Pharmacy, University of East Anglia, Norwich NR4 7TJ, UK; \\ s.gibbons@uea.ac.uk \\ 5 Department of Biological Sciences, Institute of Structural and Molecular Biology, Birkbeck, \\ University of London, Malet Street, London WC1E 7HX, UK; s.bhakta@bbk.ac.uk \\ * Correspondence: cadanquah.pharm@knust.edu.gh
}

\section{check for} updates

Citation: Danquah, C.A.; Tetteh, M.; Amponsah, I.K.; Mensah, A.Y.; Buabeng, K.O.; Gibbons, S.; Bhakta, S. Investigating Ghanaian Allium Species for Anti-Infective and Resistance-Reversal Natural Product Leads to Mitigate MultidrugResistance in Tuberculosis. Antibiotics 2021, 10, 902. https://doi.org/ 10.3390/antibiotics 10080902

Academic Editor: Manuel Simões

Received: 17 June 2021

Accepted: 15 July 2021

Published: 23 July 2021

Publisher's Note: MDPI stays neutral with regard to jurisdictional claims in published maps and institutional affiliations.

Copyright: (c) 2021 by the authors. Licensee MDPI, Basel, Switzerland. This article is an open access article distributed under the terms and conditions of the Creative Commons Attribution (CC BY) license (https:/ / creativecommons.org/licenses/by/ $4.0 /)$.

\begin{abstract}
The bulbs of Allium species are a known source of antibacterial phytochemicals. Antiinfective, efflux pump and biofilm inhibitory activities of bulb extracts of selected Ghanaian shallots Allium cepa var aggregatum were evaluated using the HT-SPOTi assay and other whole-cell phenotypic screening techniques to determine their possible mechanisms of action. Ethanol and aqueous extracts of white A. cepa inhibited the growth of Mycobacterium smegmatis mc $^{2} 155$ and Escherichia coli, respectively. The majority of the Allium extracts significantly $(p<0.05)$ exhibited efflux pump inhibitory activity against all the acid-fast, Gram-positive and Gram-negative strains used. Hexane and chloroform extract of the pink $A$. cepa and the aqueous extract of the white A. cepa significantly inhibited M. smegmatis biofilm formation. For Pseudomonas aeruginosa, the inhibition was observed at $250 \mu \mathrm{g} / \mathrm{mL}$ for the aqueous extract $(\sim 77.34 \%)$ and $125 \mu \mathrm{g} / \mathrm{mL}$ for the hexane extract $(\sim 76.51 \%)$. The results suggest that Ghanaian shallots could potentially be useful when further developed to tackle antimicrobial resistance, particularly in tuberculosis (TB).
\end{abstract}

Keywords: tuberculosis; HT-SPOTi; efflux pumps; biofilms; antimicrobial resistance

\section{Introduction}

A surge in the emergence of multidrug- and extensively-drug-resistant strains of Mycobacterium tuberculosis, has been linked to the increasing failure of antimicrobial therapy as a result of irrational use of antibiotics, and non-compliance to conventional treatment. There is, therefore, renewed research interest in natural products with the hope of discovering new antitubercular leads.

Antimicrobial resistance (AMR) has been named by the World Health Organization (WHO) as one of the three most important public health threats of the 21st century [1], and it causes persistent infections that claim millions of lives every year with enormous demands on medical and social resources. A WHO Interagency Coordination Group report on AMR estimates that 700,000 people die each year due to antibiotic resistance including 230,000 people who die from multidrug-resistant tuberculosis. This number is projected to rise to 10 million by 2050 if nothing is done to stop the AMR menace [2,3]. The overuse and misuse of antimicrobial medications, arbitrary prescribing, and unnecessary use of antibiotics in agriculture are just some of the causes of AMR. Diminishing numbers of 
new antibiotics in the drug development pipeline has intensified the urgency to develop new antimicrobials with novel modes of action or resistance-reversal mechanisms for the treatment of infections.

Biofilms and efflux pumps have been identified as key contributors to AMR. Biofilmproducing cells are known to be much less susceptible to antimicrobial agents than their planktonic forms, rendering them to be 1000-fold more resistant to antibacterial agents. Bacterial efflux pumps are membrane proteins involved in the export of antibiotics from the bacterial cell, making infections more difficult to treat [4]. Over-expression of efflux proteins in Mycobacterium species including $M$. tuberculosis plays an important role in antibiotic resistance and the development of multidrug resistance (MDR) phenotypes [5,6].

Ojah et al. (2008) [7] hypothesized that biofilm formation in M. tuberculosis could also be responsible for persistence of Mycobacterium infections. In the same work, $M$. tuberculosis and M. smegmatis biofilms were able to tolerate ten times the minimal inhibitory concentrations of the anti-tuberculosis drugs isoniazid and rifampicin [7]. The simultaneous use of efflux pump and/or biofilm inhibitors would target bacterial growth and AMR. This would therefore provide a potential way to improve the treatment of MDR infections.

A significant percentage of newly approved antibacterials are either natural products or their derivatives [8]. Additionally, hundreds of natural products possessing novel and known structural architectures have been reported to exhibit activity towards non-resistant and multidrug-resistant strains of $M$. tuberculosis. Recent research has reported the activity of plant natural products that inhibit biofilm formation and efflux pumps in bacteria $[9,10]$. Ethnopharmacological studies have shown that plants from the Amaryllidaceae family such as garlic, onions and bulbs of harmattan lily (Crinum jagus) are being used in Ghana for various respiratory tract conditions such as cough, asthma and even tuberculosis. This includes Allium sativum (bulb) and Allium cepa (bulb and leaves) [11,12]. Extracts and isolated compounds from this genus have also shown promising effects as inhibitors of efflux pumps and bacterial biofilms [13,14]. In 2018 we reported on the efflux pump inhibition and anti-biofilm activity of disulfides from Allium stipitatum and their synthetic derivatives on mycobacterial biofilms and efflux pumps [14].

In this study, we investigated the antibacterial, efflux pump and biofilm formation inhibitory activities of the bulb extracts of Ghanaian Allium cepa var aggregatum pink and white varieties (shallot) as potentially useful leads in our efforts towards the discovery and development of new anti-tuberculosis agents. Photos of Allium cepa var aggregatum white variety (a), white and pink varieties together (b) and the pink variety (c) are shown in Figure 1 respectively.

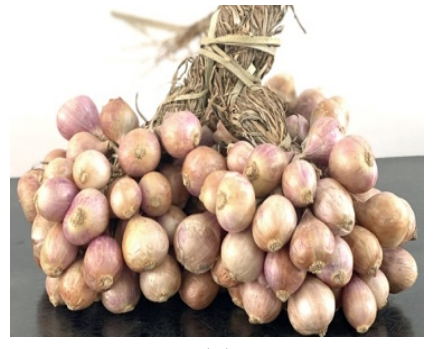

(a)

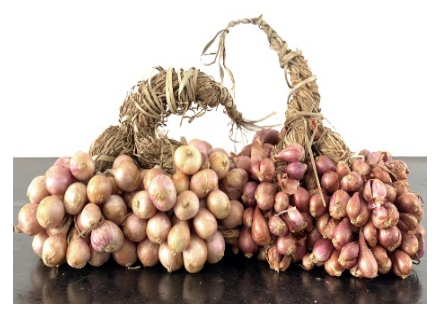

(b)

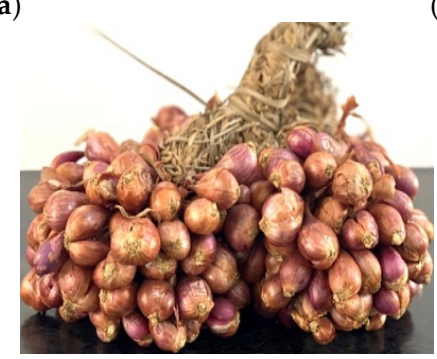

(c)

Figure 1. Photos of Allium cepa var aggregatum (White) (a); Allium cepa var aggregatum (White and Pink varieties together) (b) and Allium cepa var aggregatum (Pink) (c). 


\section{Results}

\subsection{Phytochemical Screening for the Allium Extracts}

Initial phytochemical screening confirmed the presence of various secondary metabolites including condensed tannins, alkaloids, triterpenoids, phytosterols and coumarins in majority of the extracts as shown in Table 1.

Table 1. Phytochemical screening.

\begin{tabular}{lccccccc}
\hline & MeAC & HxAC & ChAC & EtAC & AqAC & MeACb & EaAC \\
\hline Tannins & Condensed & Condensed & Condensed & Condensed & Condensed & Condensed & Condensed \\
\hline Saponins & + & - & + & + & + & + & + \\
\hline Flavonoids & + & - & + & + & + & + & + \\
\hline $\begin{array}{l}\text { Reducing } \\
\text { sugars }\end{array}$ & + & + & + & + & + & + & + \\
\hline Alkaloids & + & + & + & + & + & + & + \\
\hline Triterpenoids & + & + & + & + & + & + \\
\hline Phytosterols & + & + & + & + & + & + \\
\hline Coumarins & + & + & + & + & + & + \\
\hline
\end{tabular}

Key: + Detected. - Not detected. Allium cepa var aggregatum extracts (Pink: MeAC-methanol, HxAC-hexane, ChAC-chloroform, EtACethanol; White: AqAC-aqueous, MeACb-methanol, EaAC-ethanol).

\subsection{Allium Extracts Demonstrated Antibacterial Growth Inhibition}

The antibacterial activity of the Allium extracts was evaluated by determining the minimum inhibitory concentration (MIC) (Table 2). Extracts were moderately effective at different concentrations against the pathogens used (MIC values ranged between $250-500 \mu \mathrm{g} / \mathrm{mL}$ ). Escherichia coli was sensitive to the methanol extract of pink A. cepa, methanol and aqueous extract of white $A$. cepa with the aqueous extract of the white $A$. cepa having the highest inhibitory effect (MIC-250 $\mu \mathrm{g} / \mathrm{mL}$ ). At a concentration of $500 \mu \mathrm{g} / \mathrm{mL}$, the methanol extract of pink $A$. сеpa and ethanol and aqueous extract of white A. cepa inhibited the growth of P. mirabilis. Methanol extracts of pink A. cepa at $250 \mu \mathrm{g} / \mathrm{mL}$ and ethanol and hexane extracts at $500 \mu \mathrm{g} / \mathrm{mL}$ inhibited S. aureus. For K. pneumoniae and S. typhi, which were not sensitive to amoxicillin (MIC->500 $\mu \mathrm{g} / \mathrm{mL}$ ), only the ethanol extract of pink A. cepa was effective: K. pneumoniae (MIC-500 $\mu \mathrm{g} / \mathrm{mL}$ ) while the white $A$. cepa aqueous extract inhibited S. typhi (MIC-500 $\mu \mathrm{g} / \mathrm{mL}$ ). The P. aeruginosa was sensitive to all the extracts with the exception of the aqueous extract of the white $A$. cepa. The methanol extract of the pink $A$. cepa gave the highest activity at a concentration of $250 \mu \mathrm{g} / \mathrm{mL}$ with the other extracts at a concentration of $500 \mu \mathrm{g} / \mathrm{mL}$. In the case of S. epidermidis, the ethanol, methanol and chloroform extract of pink $A$. cepa and the methanol extract of white A. cepa had an inhibitory effect at $500 \mu \mathrm{g} / \mathrm{mL}$. The Mycobacterium strain M. smegmatis was sensitive to the ethanol extract of the pink A. cepa at a concentration of $250 \mu \mathrm{g} / \mathrm{mL}$. However, the rest of the extracts showed no activity. 
Table 2. Effect of extracts of Allium cepa on the growth of microorganisms.

\begin{tabular}{|c|c|c|c|c|c|c|c|c|}
\hline & \multicolumn{8}{|c|}{ Minimum Inhibitory Concentration MIC $(\mu \mathrm{g} / \mathrm{mL})$} \\
\hline & E. coli & P. mirabilis & S. aureus & K. pneumoniae & P. aeruginosa & S. typhi & S. epidermidis & M. smegmatis \\
\hline MeAC & 500 & 500 & 250 & $>500$ & 250 & $>500$ & 500 & $>500$ \\
\hline EtAC & $>500$ & $>500$ & 500 & 500 & 500 & $>500$ & 500 & 250 \\
\hline $\mathrm{HxAC}$ & $>500$ & $>500$ & 500 & $>500$ & 500 & $>500$ & $>500$ & $>500$ \\
\hline ChAC & $>500$ & $>500$ & $>500$ & $>500$ & 500 & $>500$ & 500 & $>500$ \\
\hline $\mathrm{MeACb}$ & 500 & $>500$ & $>500$ & $>500$ & 500 & $>500$ & 500 & $>500$ \\
\hline EaAC & $>500$ & 500 & $>500$ & $>500$ & $>500$ & $>500$ & 500 & $>500$ \\
\hline $\mathrm{AqAC}$ & 250 & 500 & $>500$ & $>500$ & 500 & 500 & 500 & $>500$ \\
\hline Ciprofloxacin & $<0.49$ & $<0.49$ & $<0.49$ & $<0.49$ & $<0.49$ & 7.81 & $<0.49$ & $<0.49$ \\
\hline Amoxicillin & 15.6 & 62.5 & 62.5 & $>500$ & 62.5 & $>500$ & 7.81 & NT \\
\hline Rifampicin & NT & NT & NT & NT & NT & NT & NT & 7.81 \\
\hline
\end{tabular}

Key: NT = Not tested. Allium cepa var aggregatum extracts (Pink: MeAC-methanol, HxAC-hexane, ChAC-chloroform, EtAC-ethanol; White: AqAC-aqueous, MeACb-methanol, EaAC-ethanol). Extracts showing activity.

\subsection{Allium Extracts Demonstrate Efflux Pump Inhibitory Effect}

The efflux pump inhibition assay was used to evaluate the activity of the extracts using ethidium bromide (EtBr) as a substrate. This assay is based on the principle that $\mathrm{EtBr}$ has a strong fluorescence property intracellularly. It binds to DNA to give an increasing fluorescence following continual accumulation. When accumulated within the bacterial cells, fluorescence intensity increases, giving an indication of the inhibition of the efflux pump mechanism within the cells [14]. In the absence of an efflux pump inhibitory activity, the cell pumps out EtBr, resulting in a low fluorescence measurement. The accumulation assay was performed with sub-MIC concentration of the extracts $\left(\frac{1}{2} \mathrm{MIC}\right)$. The results revealed that the extracts significantly ( $p$-value $<0.05$ ) enhanced the accumulation of EtBr to different levels when compared to the control with no efflux pump inhibition. This confirms their inhibitory activity against bacterial efflux pumps. The methanolic extract of the pink A. cepa was the only extract that showed no significant activity in the M. smegmatis strain (Figure 2a) ( $p$-value $>0.05)$. However, all the extracts of white $A$. cepa significantly enhanced the accumulation of EtBr (Figure 2b). All extracts significantly enhanced EtBr accumulation in P. aeruginosa (Figure 3). The effect of efflux pump inhibition in S. aureus (Figure 4a) was statistically significant for all the extracts of the pink A. cepa. Extracts of the white $A$. cepa also showed a significant inhibitory effect, with the exception of the methanol (Figure $4 \mathrm{~b}$ ). The chloroform extract of the pink $A$. cepa had a greater efflux pump inhibitory activity in the M. smegmatis and P. aeruginosa strain than the other plant extracts. The hexane extract of the pink variety of $A$. cepa is seen to have the strongest activity among the extracts in the S. aureus strain (Figure 4a). Its effect is statistically ( $p$-value $<0.05$ ) comparable to that of chlorpromazine, a known efflux inhibitor in S. aureus.

The standard efflux pump inhibitor verapamil exhibited a very good efflux pump inhibitory activity in all the bacterial cells followed by chlorpromazine. 
a.

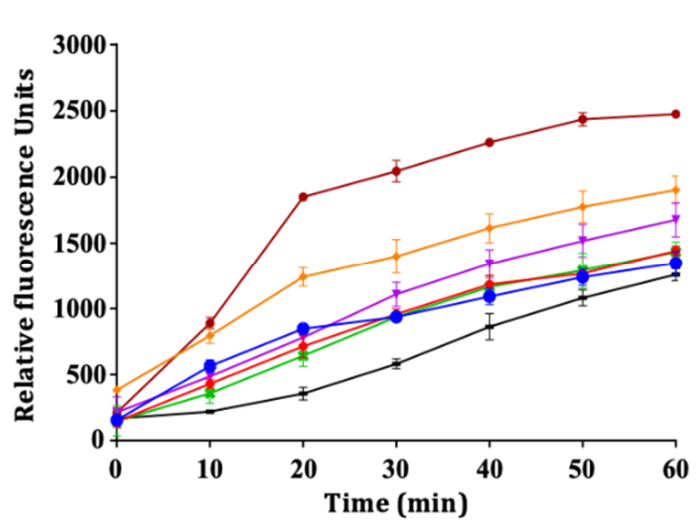

b.

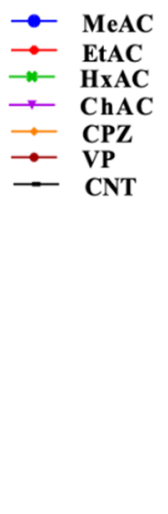

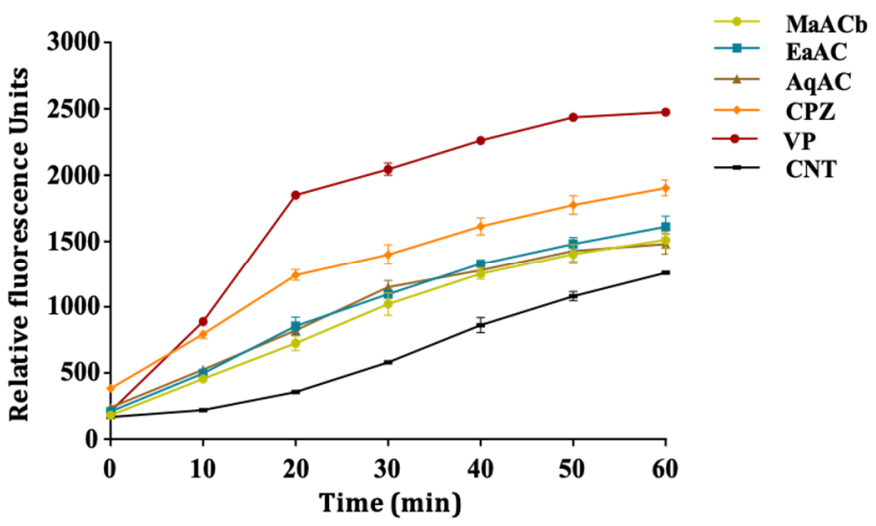

Figure 2. Effect of A. cepa extracts on the accumulation of EtBr in M. smegmatis. (a) A. cepa pink: MeAC-methanol, EtACethanol, HxAC-hexane and ChAC-chloroform; (b) Allium cepa white: MeACb-methanol, EaAC-ethanol, AqAC-aqueous, CPZ-chlorpromazine; VP-verapamil; CNT-control.

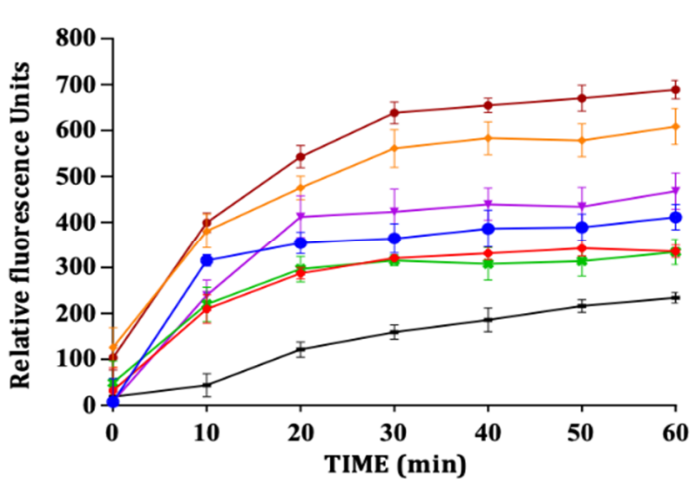

b.

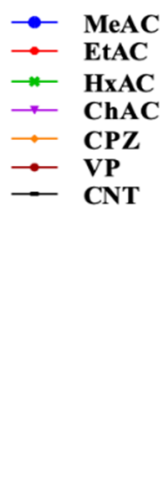

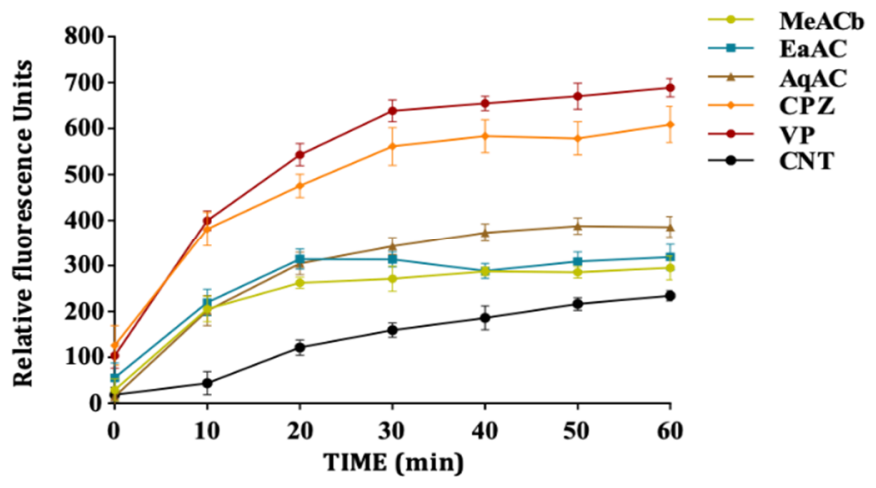

Figure 3. Effect of A. cepa on the accumulation of EtBr in P. aeruginosa. (a) A. cepa pink: MeAC-methanol, EtAC-ethanol, HxAC-hexane and ChAC-chloroform; (b) Allium cepa white: MeACb-methanol, EaAC-ethanol, AqAC-aqueous, CPZchlorpromazine; VP-verapamil; CNT-control.

a

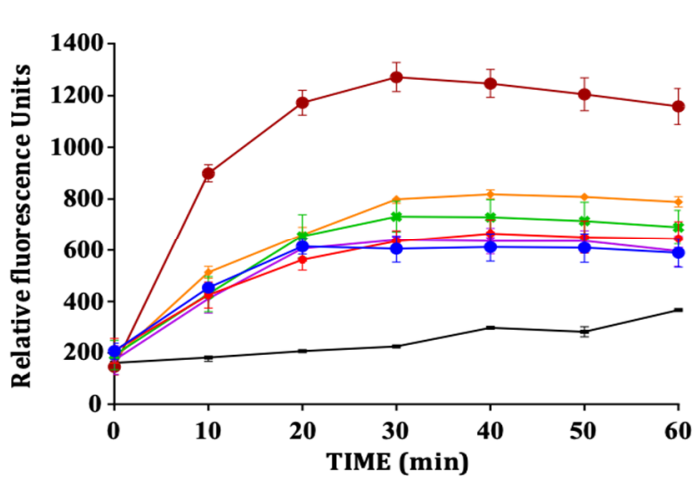

b.

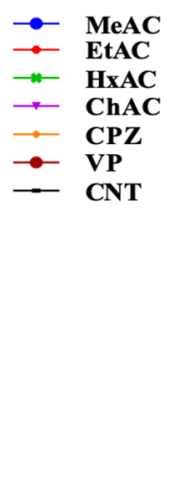

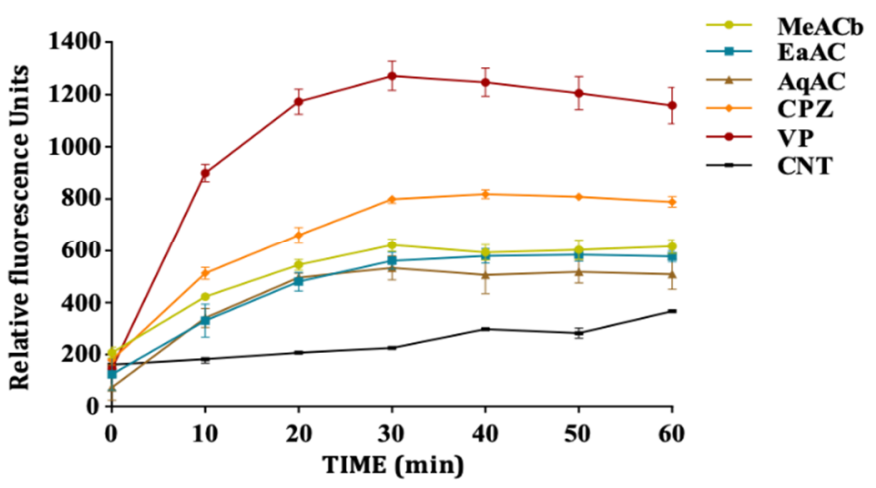

Figure 4. Effect of A. cepa on the accumulation of EtBr in S. aureus. (a) A. cepa pink: MeAC-methanol, EtAC-ethanol, HxAC-hexane and ChAC-chloroform; (b) Allium cepa white: MeACb-methanol, EaAC-ethanol, AqAC-aqueous, CPZchlorpromazine; VP-verapamil; CNT-control. 


\subsection{Biofilm Formation}

The result from the crystal violet staining assay showed that biofilm formation was high in the TSB medium supplemented with additional glucose to $1 \%$ than the medium without glucose supplement (Figure 5). Similar results were observed in the microtitre plate method (results not shown). For better biofilm formation we recommend that TSB be supplemented with additional glucose.

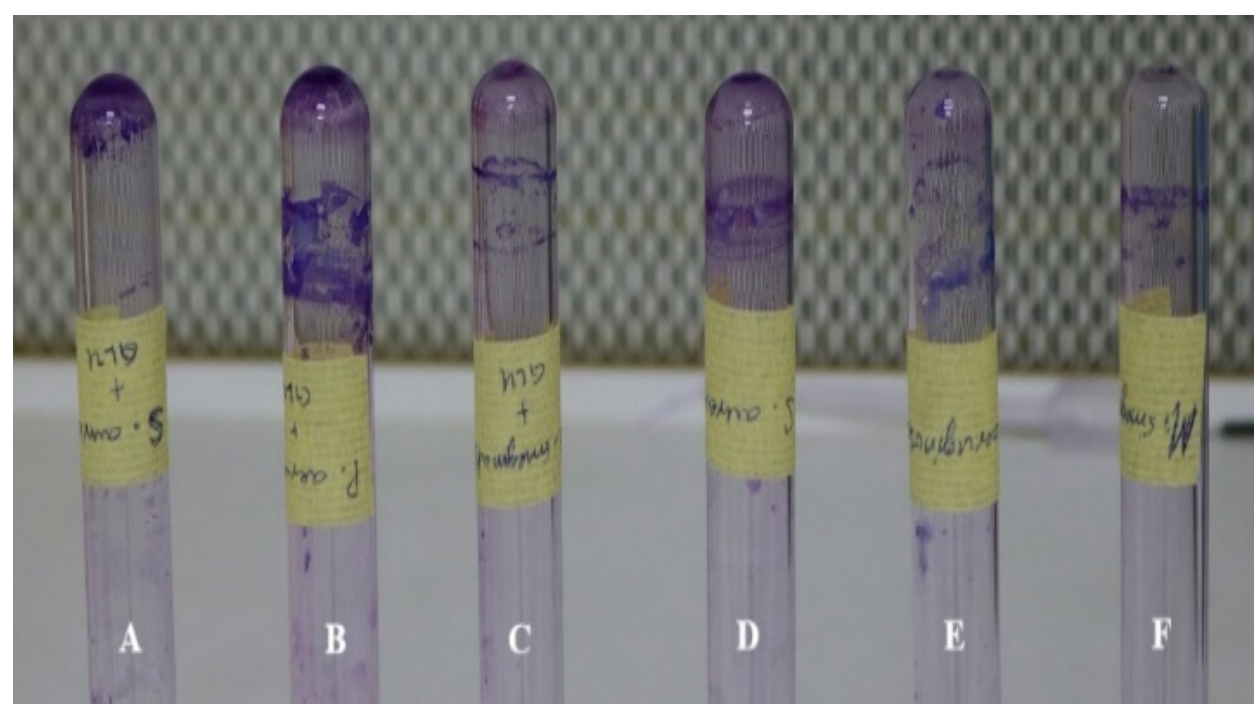

Figure 5. Biofilm formation by (A) S. aureus + glucose; (B) P. aeruginosa + glucose; (C) M. smegmatis + glucose; (D) S. aureus; (E) P. aeruginosa; (F) M. smegmatis.

\subsection{Allium Extracts Demonstrate Biofilm Inhibition}

Biofilm formation contributes to bacterial persistence, which contributes to antimicrobial resistance. Hence, the A. cepa extracts were evaluated to find out if they have a biofilm inhibitory effect. The different Allium extracts showed varying levels of biofilm inhibition at different concentrations. The biofilm inhibition of the extracts on the P. aeruginosa and $M$. smegmatis biofilms were concentration-dependent. A good inhibition of biofilm formation was observed for $M$. smegmatis. Compared to the other extracts, the hexane and chloroform extract of the pink A. cepa and the aqueous extract of the white A. cepa had significant inhibition on the M. smegmatis biofilm (Figure 6A). The hexane extract gave the highest inhibitory effect in all the concentrations used. The aqueous extract of the white A. cepa and the hexane extract of the pink $A$. cepa had a greater inhibitory effect in $P$. aeruginosa biofilm formation (Figure 6B). The highest inhibition was observed at $250 \mu \mathrm{g} / \mathrm{mL}$ for the aqueous extract $(\sim 77.34 \%)$ and $125 \mu \mathrm{g} / \mathrm{mL}$ for the hexane extract $(\sim 76.51 \%)$.

All the other extracts moderately inhibited biofilm formation in P. aeruginosa at concentrations of $125 \mu \mathrm{g} / \mathrm{mL}$ and $250 \mu \mathrm{g} / \mathrm{mL}$. Unlike the M. smegmatis and P. aeruginosa strains, the $S$. aureus strain was less susceptible to biofilm inhibition by the extracts. However, the hexane extract of pink $A$. cepa was significantly effective at inhibiting $S$. aureus biofilms ( 69.3\%). The aqueous extract of white $A$. cepa was also effective at inhibiting biofilm formation at a sub-inhibitory concentration of $62.5 \mu \mathrm{g} / \mathrm{mL}(\sim 66.8 \%)$ (Figure $6 \mathrm{C}$ ). 
A.

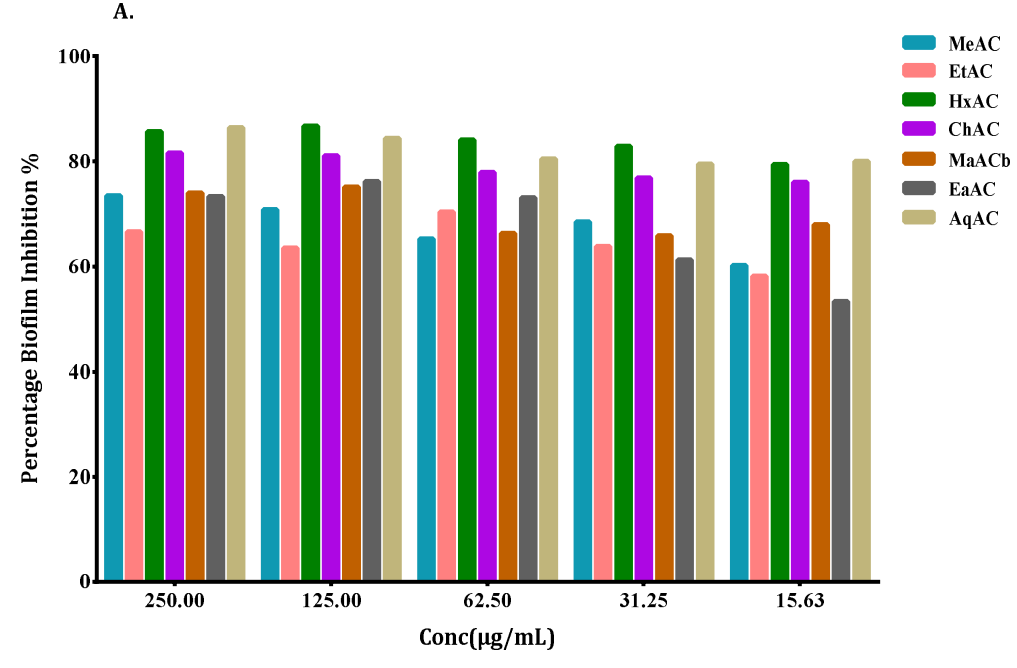

B.

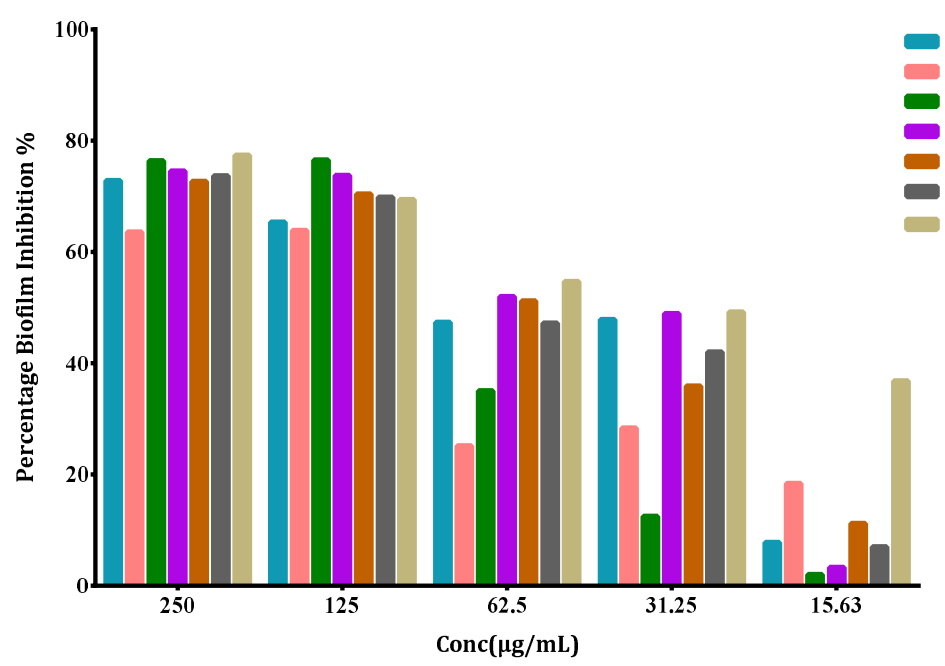

c.

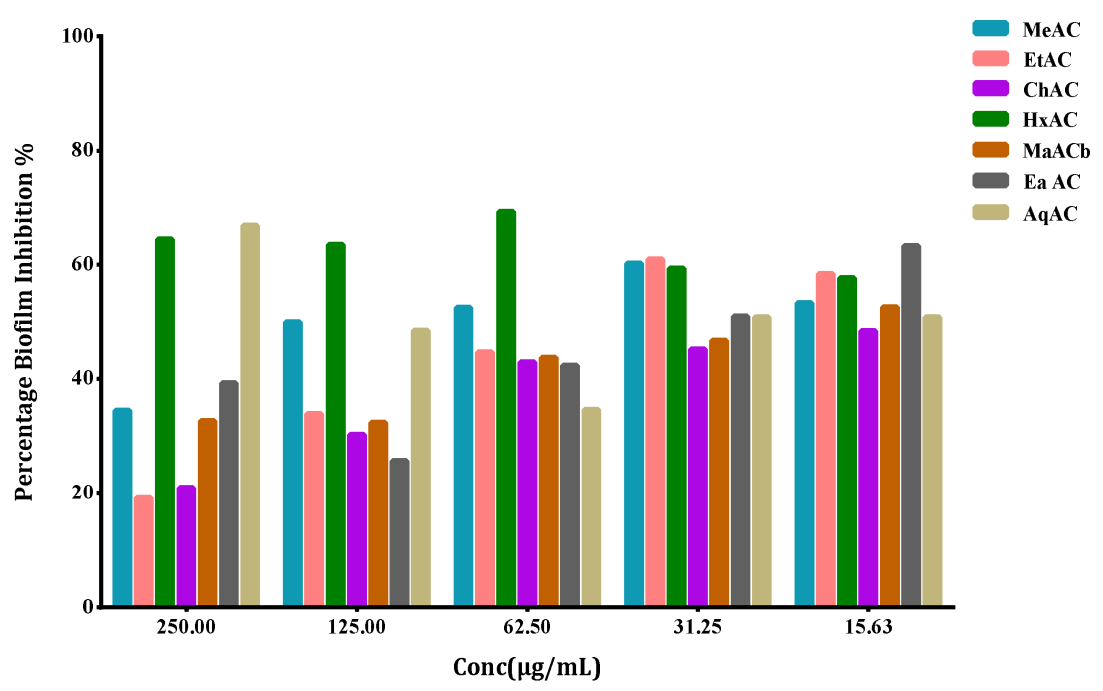

Figure 6. Effects of A. cepa extracts on biofilm formation in (A) M. smegmatis (B) P. aeruginosa (C) S. aureus strains expressed as percentage (\%) inhibition and evaluated by the crystal violet staining assay. Values are the average of at least three independent determinations. Extracts of $A$. cepa pink: MeAC-methanol, EtAC-ethanol, HxAC-hexane and ChAC-chloroform; Allium cepa white: MeACb-methanol, EaAC-ethanol, AqAC-aqueous, CPZ-chlorpromazine; VP-verapamil; CNT-control. 


\section{Discussion}

The minimum inhibitory concentration determined from the HT-SPOTi assay showed that extracts of both white and pink $A$. cepa had a broad spectrum of activity on the Grampositive and Gram-negative strains at different concentrations. Methanolic and ethanolic extracts of the pink $A$. cepa and the aqueous extract of the white $A$. cepa showed high activity on most of the pathogens. Overall, P. aeruginosa was more sensitive to all the extracts with the exception of the aqueous extract of the white $A$. cepa. The methanol extract of the pink $A$. cepa gave the highest activity (MIC- $250 \mu \mathrm{g} / \mathrm{mL}$ ) while the other extracts (MIC-500 $\mu \mathrm{g} / \mathrm{mL}$ ). The aqueous extract was the most effective among the white A. cepa, inhibiting four pathogens, MIC- $250 \mu \mathrm{g} / \mathrm{mL}$ for E. coli and $500 \mu \mathrm{g} / \mathrm{mL}$ for P. mirabilis, P. aeruginosa and S. typhi, respectively. Ethanolic extract of white $A$. cepa had no activity on the pathogens except for P. mirabilis $(500 \mu \mathrm{g} / \mathrm{mL})$. In as much as K. pneumoniae and P. mirabilis are intrinsically insensitive to antibiotics, it is worth noting that some extracts inhibited their growth at $500 \mu \mathrm{g} / \mathrm{mL}$. Only the ethanol extract of the white $A$. cepa inhibited the growth of M. smegmatis with an MIC of $250 \mu \mathrm{g} / \mathrm{mL}$; even though the activity is moderate, this highlights the need to further explore the extract as a potential antimycobacterial agent since the treatment of mycobacterial infections (notably M. tuberculosis) is being threatened by multidrug-resistant strains [6].

In the bacterial efflux pump inhibition assay, the majority of the Allium extracts significantly enhanced the accumulation of ethidium bromide in all the strains when compared to the control with no drug. The results suggest that $A$. cepa extracts possess an efflux pump inhibitory activity, hence interfering with ethidium bromide efflux from the bacteria cells that express multiple efflux proteins. This indicates their potential as efflux pump inhibitors. Similar results of efflux pump inhibition by Allium plants have been reported; Extracts of A. sativum and allyl sulfide (a bioactive component of most Allium plants) have been shown to inhibit the multidrug efflux pump EmrD-3 of $V$. cholerae [13]. Disulfides from A. stipitatum have also been found to have efflux pump inhibitory activity against Mycobacterium strains [14]. Interestingly, some of the Allium extracts, which did not show antimicrobial activity (MIC $>500 \mu \mathrm{g} / \mathrm{mL}$ ) especially in M. smegmatis, were able to inhibit efflux pump activity. These further support the claims that plant extracts are excellent candidates for lead optimization of efflux pump inhibitors [15]. In general, it was observed that the less polar solvent extracts had good activity than the more polar extracts. Drug efflux by efflux pumps is a contributing factor in the growing problem of antimicrobial resistance contributing to the intrinsic and acquired resistance in a wide variety of bacteria. This keeps complicating the treatment of infectious diseases [16,17]. Therefore, the Ghanaian Allium cepa var aggregatum plant demonstrating to have efflux pump inhibitory properties could have the potential of restoring the bacterial susceptibility to drugs and enhance the treatment of infectious diseases. Further work is underway to isolate and identify the bioactive compounds and also ascertain the modulatory effect of these extracts on drugs in clinical use.

Bacterial biofilms have contributed greatly to the persistence of antimicrobial resistance. They have also become a major leading cause of multi-drug resistance. In the present report, the different extracts of white and pink A. cepa varieties inhibited Gram-positive, Gram-negative and Mycobacterium biofilms at sub-lethal concentrations. The ability of each extract to inhibit biofilm formation was dependent on the type of solvent and the concentration of the extract used. Overall, non-polar hexane extract of the pink variety of $A$. сеpa and the aqueous extract of the white variety inhibited biofilm formation in all strains of bacteria. The biofilm inhibition activity of the extracts against $P$. aeruginosa and $M$. smegmatis were dose-dependent. The underlying mechanisms of the antibiofilm properties of these extracts could be due to, inhibiting the formation of the polymer matrix, suppressing cell adhesion and attachment, interrupting extracellular matrix generation and / or decreasing virulence factors production. These mechanisms have accounted for biofilm inhibition properties of natural products according to Lu et al. in 2019 [18]. In recent years, the antibiofilm properties of Allium species have attracted attention. For 
example, the biofilm inhibition properties of A. sativum, A. stipitatum, A. orientale and A. porrum have been documented [19-22]. The inhibition of bacterial efflux pumps has also been suggested as a strategy to inhibit biofilm formation [23].

It is, therefore, noteworthy that the Ghanaian A. cepa var aggregatum extracts have demonstrated both efflux pump and biofilm inhibitory properties which are interesting since a major challenge in the management and emergence of multidrug-resistant tuberculosis is the long duration of treatment. Expression of efflux proteins and biofilm formation has been recognized as a common mechanism contributing to both virulence and drug tolerance in M. tuberculosis [24]. Richards and Ojha (2014) [25] reported that Mycobacterium biofilms enhance the spontaneous growth of mycobacteria, particularly $M$. tuberculosis, and also promotes persistence against exogenous threats. This confers drug resistance to drug-susceptible M. tuberculosis mutants [25].

The ability of the extracts to inhibit efflux pumps and biofilm formation in M. smegmatis indicates their potential to mitigate M. tuberculosis by their pleiotropic mode of action or by their ability to possibly reverse resistance by improving sensitivity when combined with existing antibacterials that have lost their sensitivity due to antibiotic resistance.

\section{Materials and Methods}

\subsection{Materials}

Chlorpromazine, verapamil, rifampicin, ciprofloxacin, EtBr and phosphate-buffered saline (PBS) tablets $(0.01 \mathrm{M}$ phosphate buffer, $0.0027 \mathrm{M}$ potassium chloride and $0.137 \mathrm{M}$ sodium chloride, $\mathrm{pH}$ 7.4) were purchased from Sigma-Aldrich, St Louis, MO, USA. Middlebrook 7H10 agar, Middlebrook 7H9 broth, oleic acid-albumin-dextrose-catalase (OADC) supplement and albumin-dextrose-catalase (ADC) supplement were purchased from Difco, Franklin Lakes, NJ, USA. Tryptic soy broth (TSB) (HiMedia, Mumbai, India) was used for the anti-biofilm assay. All solutions were prepared in distilled water or DMSO on the day of the experiment.

\subsection{Collection of Plant Material and Extraction}

The pink and white varieties of the bulbs of Ghanaian shallot (Allium cepa var aggregatum) were purchased from Keta in the Volta region of Ghana ( $\left.5^{\circ} 55^{\prime} 19.5384^{\prime \prime} \mathrm{N} 0^{\circ} 59^{\prime} 31.8948^{\prime \prime} \mathrm{E}\right)$ on 13 April 2019. Dr. George Henry Sam, Department of Herbal Medicine, KNUST, Ghana authenticated the plant materials, and voucher specimens (Pink Allium cepa var aggregatum-KNUST /2019/HM1/013; White Allium cepa var aggregatum-KNUST/2019/HM1/ 014) were kept in the Herbarium. The fresh bulbs were washed thoroughly and blended with an Akai (Tokyo, Japan) electric blender (Model no. BD033A SO4M) using hexane, chloroform, ethanol, methanol and water as solvents. The extracts were filtered, and the solvents evaporated using a rotary evaporator (Buchi Rotavapor R-210, BÜCHI Labortechnik AG CH-9230 Flawil 1, Switzerland) to yield semi-solid dark brown extracts. All the extracts were stored in a fridge at $-4{ }^{\circ} \mathrm{C}$ until used.

\subsection{Microbial Strains and Culture Conditions}

The following microbial strains were used in this study: Escherichia coli (ATCC 25922), Staphylococcus aureus (ATCC 25923), Pseudomonas aeruginosa (ATCC 4853); clinical strains of Salmonella typhi, Klebsiella pneumoniae, Staphylococcus epidermidis, Proteus mirabilis; and Mycobacterium smegmatis mc $^{2} 155$ (ATCC 19420), a fast-growing mycobacteria used as a surrogate for $M$. tuberculosis. The pathogens were obtained from the Department of Pharmaceutical Microbiology, and the Cell Culture Laboratory, Department of Pharmacology, KNUST. Glycerol stocks of the microorganisms were stored at $-80^{\circ} \mathrm{C}$. Nutrient agar (Oxoid, Basingstoke, England) was used to culture the Gram-positive and Gram-negative bacteria and fungus while Middlebrook 7H10 agar supplemented with OADC was used for M. smegmatis. 


\subsection{Methods}

\subsubsection{Antimicrobial Susceptibility Assay with HT-SPOTi}

The minimum inhibitory concentrations (MIC) of the different extracts of cepa varieties were determined using the high-throughput spot culture growth inhibition (HT-SPOTi) assay as described by Danquah et al. (2016) [26]. Two-fold serial dilutions of a stock concentration of the extracts $(50 \mathrm{mg} / \mathrm{mL})$ were done in a PCR half-skirted 96-well plate to give a concentration range of $50-0.05 \mathrm{mg} / \mathrm{mL}$. A volume $(2 \mu \mathrm{L})$ of the drug dilution was transferred into a corresponding 96-well plate and $200 \mu \mathrm{L}$ of molten agar was dispensed into it with shaking (nutrient agar was used for the bacteria and fungus, and Middlebrook 7H10 agar with $0.5 \%(v / v)$ glycerol supplemented with $10 \%$ OADC for the Mycobacterium strain). The plate was left to solidify. A volume of $2 \mu \mathrm{L}$ of the bacterial suspension containing $\sim 1 \times 10^{6} \mathrm{cfu} / \mathrm{mL}$ was spotted onto each well of the 96 -well plate. The bacterial spot suspension was absorbed into the agar (approximately $5 \mathrm{~min}$ ). The plate was sealed with parafilm, wrapped with aluminum foil and incubated at $37^{\circ} \mathrm{C}$ for $18-48 \mathrm{~h}$ based on the doubling time of the bacterial models used in this study. Wells with no drug were added to serve as growth control. Ciprofloxacin, a broad-spectrum antibiotic of the fluoroquinolone class, which is bactericidal and inhibits topoisomerase II (DNA gyrase) and amoxicillin, a broad-spectrum penicillin which is bactericidal and inhibits bacterial cell wall synthesiswere used as drug control for Gram-positive and Gram-negative bacteria. Rifampicin, a first-line TB drug was used for the acid-fast bacteria, Mycobacterium. The minimum inhibitory concentration (MIC) was defined as the lowest concentration of antimicrobial agent that inhibited visible bacterial growth within the incubation period. The test was done in triplicate.

\subsubsection{Efflux Pump Inhibitory Assay}

The whole-cell phenotypic efflux pump inhibition assay was performed according to previously published protocols with some modification using M. smegmatis, S. aureus and $P$. aeruginosa [27-30]. The Gram-positive and Gram-negative cells were cultured in $10 \mathrm{~mL}$ nutrient broth while the Mycobacterium was cultured in Middlebrook 7H9 broth (Difco, USA) in Erlenmeyer flasks containing 10\% OADC enrichment (Becton Dickinson, Franklin Lakes, NJ, USA) and $0.05 \%$ Tween 80 . They were cultured at $37^{\circ} \mathrm{C}$ with shaking at $150 \mathrm{rpm}$ until an optical density of $600 \mathrm{~nm}\left(\mathrm{OD}_{600}\right)$ of $0.8-1$ (mid-log phase). The $\mathrm{OD}_{600}$ of the cultures was adjusted to 0.4 after which $10 \mathrm{~mL}$ was centrifuged at $3000 \mathrm{rpm}$ for $10 \mathrm{~min}$. The supernatant was discarded, and pellets washed with sterile PBS.

The pellets were re-suspended in $10 \mathrm{~mL}$ of sterile PBS. The bacteria suspension $(500 \mu \mathrm{L})$ (Test) and $500 \mu \mathrm{L}$ of PBS (Blank) were pipetted into $2 \mathrm{~mL}$ Eppendorf tubes and glucose added to a final concentration of $0.4 \%$. Different concentrations of the extracts and compounds were added at half their MIC's. The suspension was vortexed to distribute the cells uniformly. Aliquots of $100 \mu \mathrm{L}$ from each mixture were transferred into 96-well plates (in triplicates) and $5 \mu \mathrm{L}$ of EtBr added to give a final concentration of $0.5 \mathrm{mg} / \mathrm{L}$. The EtBr was added just before reading the fluorescence intensity. The fluorescence was measured using the microplate reader (Biotek Synergy H1 Hybrid Multi-Mode Reader: 271230, Vermont, VT, USA) at $530 \mathrm{~nm}$ (excitation) and $585 \mathrm{~nm}$ (emission) every $10 \mathrm{~min}$ over a period of $60 \mathrm{~min}$ at $37^{\circ} \mathrm{C}$. This measures the effect of the extracts on the accumulation of EtBr within the cells by inhibiting the efflux pumps. The known efflux pump inhibitors, verapamil and chlorpromazine, served as reference drugs and a drug-free culture was used as control.

\subsubsection{Biofilm Inhibition Assay}

Evaluation of Biofilm Forming Potential

The biofilm-forming ability of M. smegmatis, P. aeruginosa and S. aureus was evaluated by the tube method [31] and the microtitre-plate method [32] with minor modifications described below. 


\section{Tube Method}

The tube method is a qualitative assay for the detection of microorganisms that produce biofilm. It is based on the occurrence of a visible film adhering to the surface of the tube. As described by Christensen et al. in 1985 [31], bacterial isolates were inoculated in a polystyrene test tube that contained $5 \mathrm{~mL}$ of TSB and incubated at $37^{\circ} \mathrm{C}$ for $24 \mathrm{~h}$.

Cultures supplemented with glucose to a concentration of $1 \%$ was included for comparison. The contents were decanted, the tubes washed twice with phosphate-buffered saline and left to dry. Subsequently, the polystyrene tubes were stained with crystal violet $(0.1 \%)$ for $15 \mathrm{~min}$, rinsed with PBS and air-dried in an inverted position. The occurrence of visible film lined the walls, and the bottom of the tube indicates biofilm production [31]. The tests were carried out in triplicate.

\section{Microtitre-Plate Method}

The biofilm-forming potential of the microorganisms was quantitatively analyzed using the microtitre-plate biofilm formation assay as previously described with some modifications [32-34]. Briefly, a loopful of an overnight culture of the microorganisms were inoculated in $5 \mathrm{~mL}$ Tryptone Soy Broth (TSB) and incubated at $37^{\circ} \mathrm{C}$ for $24 \mathrm{~h}$ with shaking at $150 \mathrm{rpm}$. The cultures were 1:100 diluted in TSB (supplemented with additional glucose to a final concentration of $1 \%$ ) after incubation, and $100 \mu \mathrm{L}$ of each diluted culture pipetted into a 96-well flat-bottom microtitre plate (Star Lab, Humberg, Germany). Plates were covered and incubated at $37^{\circ} \mathrm{C}$. After $24 \mathrm{~h}$, the planktonic cells were aspirated, and wells were washed with PBS to remove planktonic bacteria.

Adherent bacterial biofilms were fixed by drying in the incubator for $30 \mathrm{~min}$ and stained with $125 \mu \mathrm{L}$ of $0.1 \%(w / v)$ crystal violet (Burgoyne Burbidges and Co, Mumbai, India) for $10 \mathrm{~min}$ at room temperature. Excess stain was washed with distilled water and left to air dry. Subsequently, the stain was solubilized with $125 \mu \mathrm{L}$ of $95 \%$ ethanol and left for $10 \mathrm{~min}$. The optical density of each well was measured at $600 \mathrm{~nm}\left(\mathrm{OD}_{600}\right)$ using an automated plate reader (Biotek Synergy H1 Hybrid Multi-Mode Reader: 271230).

\section{Inhibition of Biofilm Formation}

The ability of the Allium extracts to inhibit biofilm formation was measured using the microtiter plate-based assay as described. A volume $(180 \mu \mathrm{L})$ of each diluted culture was pipetted into a 96-well flat-bottom microtitre plate. Two-fold serial dilutions of the extracts were made with Tryptic soy broth (TSB) to achieve sub-minimum inhibitory concentrations ranging from $250-15.63 \mu \mathrm{g} / \mathrm{mL}$. An amount $(20 \mu \mathrm{L})$ of the extract's solution was then pipetted into each of the 96-well plates. The plates were incubated undisturbed for $24 \mathrm{~h}$ at $37^{\circ} \mathrm{C}$. Wells containing only microorganisms were included to serve as the growth control. After $24 \mathrm{~h}$ of incubation at $37^{\circ} \mathrm{C}$, the supernatant was collected and the plates were washed, fixed and stained as described above. The optical density of each well was measured at $600 \mathrm{~nm}\left(\mathrm{OD}_{600}\right)$ using an automated plate reader. The bioassay was performed in duplicate. The mean absorbance of the samples was determined, and the percentage inhibition of biofilm was calculated using the equation:

$$
\text { Percentage Biofilm inhibition }(\%)=\left(\frac{\mathrm{OD}_{\text {negative control }-\mathrm{OD}_{\text {Experimental }}}}{\mathrm{OD}_{\text {negative control }}}\right) \times 100
$$

\subsection{Statistical Analysis}

GraphPad prism 8.0 software was used for the statistical analysis using One-Way Analysis of Variance (ANOVA). Results were quantified as mean \pm SEM. Statistical differences between mean values were performed with Dunnett's Multiple Comparison Test at $p<0.05$. 


\section{Conclusions}

The findings from this study showed that the bulbs of Ghanaian Allium cepa var aggregatum (shallots) are a potential source of phytochemical hits to tackle antimicrobial resistance as a result of their inhibition of whole-cell efflux pumps, biofilm formation and antibacterial selectivity against $M$. smegmatis resistance mechanisms. Bioactivity-guided isolation and characterization of compounds from these species could lead to the discovery of lead bioactive compounds that can be developed as drugs for clinical use.

Author Contributions: Conceptualization and design of research work C.A.D.; methodology, S.B., C.A.D., M.T.; validation, S.G., A.Y.M. and S.B.; formal analysis, C.A.D., I.K.A., S.G., A.Y.M., S.B.; investigation, M.T., C.A.D.; resources, C.A.D., K.O.B.; data curation, M.T., C.A.D., S.G., S.B.; Writingoriginal draft preparation M.T., C.A.D.; writing-review and editing, S.G., S.B., A.Y.M., I.K.A., K.O.B.; supervision, C.A.D.; project administration, C.A.D.; funding acquisition, C.A.D., I.K.A., A.Y.M., K.O.B. All authors have read and agreed to the published version of the manuscript.

Funding: This research was funded by Kwame Nkrumah University of Science and Technology (KNUST) Interdisciplinary Research Fund (KREF) to CAD. SB acknowledges the Global Challenges Research Fund (GCRF QR 105123-21) for the support in building a UK-Ghana capacity for tackling antimicrobial drug resistance in tuberculosis.

Data Availability Statement: The data used to support the findings of the study can be made available upon request through the corresponding author.

Acknowledgments: The authors would like to thank R.C. Abaidoo, Office of Grants and Research, KNUST for his immense support towards this work. Clifford Asare, Herbal Technologist, Department of Herbal Medicine, KNUST; Prince Dagadu, Laboratory Technologist at the Cell Culture Laboratory: and all members of the Drug Discovery Team (Selase Ativui, Peace Doe, Michael Ofori, Williams Asamoah Adu), Department of Pharmacology, Faculty of Pharmacy and Pharmaceutical Sciences, College of Health Sciences, KNUST are duly acknowledged.

Conflicts of Interest: The authors declare no conflict of interest.

\section{References}

1. World Health Organization (WHO). Antimicrobial Resistance: Global Report on Surveillance 2014; World Health Organization: Geneva, Switzerland, 2014; ISBN 9789241564748.

2. Interagency Coordination Group (IACG). No Time to Wait: Infections from Drug-Resistant Securing the Future from Drug-Resistant Infections; Artforum International Magazine: New York, NY, USA, 2019.

3. Bretagne, G.; O'Neill, J. Antimicrobial Resistance: Tackling a Crisis for the Health and Wealth of Nations; Review on Antimicrobial Resistance: London, UK, 2014.

4. McKeegan, K.S.; Borges-Walmsley, M.I.; Walmsley, A.R. The structure and function of drug pumps: An update. Trends Microbiol. 2003, 11, 21-29. [CrossRef]

5. Rossi, E.D.; Aínsa, J.A.; Riccardi, G. Role of mycobacterial efflux transporters in drug resistance: An unresolved question. FEMS Microbiol. Rev. 2006, 30, 36-52. [CrossRef]

6. Viveiros, M.; Leandro, C.; Amaral, L. Mycobacterial efflux pumps and chemotherapeutic implications. Int. J. Antimicrob. Agents 2003, 22, 274-278. [CrossRef]

7. Ojha, A.K.; Baughn, A.D.; Sambandan, D.; Hsu, T.; Trivelli, X.; Guerardel, Y.; Alahari, A.; Kremer, L.; Jacobs, W.R., Jr.; Hatfull, G.F Growth of Mycobacterium tuberculosis biofilms containing free mycolic acids and harbouring drug-tolerant bacteria. Mol. Microbiol. 2008, 69, 164-174. [CrossRef] [PubMed]

8. Brown, D.G.; Lister, T.; May-Dracka, T.L. New natural products as new leads for antibacterial drug discovery. Bioorganic Med. Chem. Lett. 2014, 24, 413-418. [CrossRef] [PubMed]

9. Karbasizade, V.; Dehghan, P.; Sichani, M.M.; Shahanipoor, K.; Sepahvand, S.; Jafari, R.; Yousefian, R. Evaluation of three plant extracts against biofilm formation and expression of quorum sensing regulated virulence factors in Pseudomonas aeruginosa. Pak. J. Pharm. Sci. 2017, 3, 585-589.

10. Shriram, V.; Khare, T.; Bhagwat, R.; Shukla, R.; Kumar, V. Inhibiting bacterial drug efflux pumps via phyto-therapeutics to combat threatening antimicrobial resistance. Front. Microbiol. 2018, 9, 2990. [CrossRef]

11. Nguta, J.M.; Appiah-Opong, R.; Nyarko, A.K.; Yeboah-Manu, D.; Addo, P.G.A. Medicinal plants used to treat TB in Ghana. Int. J. Mycobacteriol. 2015, 4, 116-123. [CrossRef] [PubMed]

12. Minkah, P.A.B.; Danquah, C.A. Anti-infective, anti-inflammatory and antipyretic activities of the bulb extracts of Crinum jagus (J. Thomps.) Dandy (Amaryllidaceae). Sci. Afr. 2021, 12, e00723. [CrossRef] 
13. Bruns, M.M.; Kakarla, P.; Floyd, J.T.; Mukherjee, M.M.; Ponce, R.C.; Garcia, J.A.; Ranaweera, I.; Sanford, L.M.; Hernandez, A.J.; Willmon, M.T.; et al. Modulation of the multidrug efflux pump EmrD-3 from Vibrio cholerae by Allium sativum extract and the bioactive agent allyl sulfide plus synergistic enhancement of antimicrobial susceptibility by A. sativum extract. Arch. Microbiol. 2017, 99, 1103-1112. [CrossRef]

14. Danquah, C.A.; Kakagianni, E.; Khondkar, P.; Maitra, A.; Rahman, M.; Evangelopoulos, D.; McHugh, T.D.; Stapleton, P.; Malkinson, J.; Bhakta, S.; et al. Analogues of Disulfides from Allium stipitatum demonstrate potent anti-tubercular activities through drug efflux pump and biofilm inhibition. Sci. Rep. 2018, 8, 1150. [CrossRef] [PubMed]

15. Stavri, M.; Piddock, L.J.V.; Gibbons, S. Bacterial efflux pump inhibitors from natural sources. J. Antimicrob. Chemother. 2007, 59, 1247-1260. [CrossRef] [PubMed]

16. Li, X.Z.; Nikaido, H. Efflux-mediated drug resistance in bacteria: A n update. Drugs 2009, 69, 1555-1623. [CrossRef]

17. Schindler, B.D.; Kaatz, G.W. Multidrug efflux pumps of Gram-positive bacteria. Drug Resist. Updates 2016, 27, 1-3. [CrossRef]

18. Lu, L.; Hu, W.; Tian, Z.; Yuan, D.; Yi, G.; Zhou, Y.; Cheng, Q.; Zhu, J.; Li, M. Developing natural products as potential anti-biofilm agents. Chin. Med. 2019, 14, 1-17. [CrossRef]

19. Mohsenipour, Z. The effects of Allium sativum extracts on biofilm formation and activities of six pathogenic bacteria. Jundishapur J. Microbiol. 2015, 8. [CrossRef] [PubMed]

20. Pellegrini, M.C.; Ponce, A.G. Beet (Beta vulgaris) and Leek (Allium porrum) Leaves as a Source of Bioactive Compounds with Anti-quorum Sensing and Anti-biofilm Activity. Waste Biomass Valorization 2019, 1-9. [CrossRef]

21. Ceylan, O.; Alic, H. Antibiofilm, antioxidant, antimutagenic activities and phenolic compounds of Allium orientale BOISS. Braz. Arch. Biol. Technol. 2015, 58, 935-943. [CrossRef]

22. Karunanidhi, A.; Ghaznavi-Rad, E.; Hamat, R.A.; Pichika, M.R.; Lung, L.T.T.; Mohd Fauzi, F.; Chigurupati, S.; van Belkum, A.; Neela, V. Antibacterial and Antibiofilm Activities of Nonpolar Extracts of Allium stipitatum Regel. against Multidrug Resistant Bacteria. Biomed. Res. Int. 2018. [CrossRef]

23. Kvist, M.; Hancock, V.; Klemm, P. Inactivation of efflux pumps abolishes bacterial biofilm formation. Appl. Environ. Microbiol. 2008, 74, 7376-7382. [CrossRef]

24. Szumowski, J.D.; Adams, K.N.; Edelstein, P.H.; Ramakrishnan, L. Antimicrobial efflux pumps and Mycobacterium tuberculosis drug tolerance: Evolutionary considerations. Curr. Top. Microbiol. Immunol. 2012, 81-108. [CrossRef]

25. Richards, J.P.; Ojha, A.K. Mycobacterial Biofilms. Microbiol. Spectr. 2014. [CrossRef] [PubMed]

26. Danquah, C.A.; Maitra, A.; Gibbons, S.; Faull, J.; Bhakta, S. HT-SPOTi: A rapid drug susceptibility test (DST) to evaluate antibiotic resistance profiles and novel chemicals for anti-infective drug discovery. Curr. Protoc. Microbiol. 2016. [CrossRef]

27. Rodrigues, L.; Wagner, D.; Viveiros, M.; Sampaio, D.; Couto, I.; Vavra, M.; Kern, W.V.; Amaral, L. Thioridazine and chlorpromazine inhibition of ethidium bromide efflux in Mycobacterium avium and Mycobacterium smegmatis. J. Antimicrob. Chemother. $2008,61$. [CrossRef]

28. Lechner, D.; Gibbons, S.; Bucar, F. Plant phenolic compounds as ethidium bromide efflux inhibitors in Mycobacterium smegmatis. J. Antimicrob. Chemother. 2008, 62, 345-348. [CrossRef]

29. Prasch, S.; Duran, A.G.; Chinchilla, N.; Molinillo, J.M.G.; Macías, F.A.; Bucar, F. Resistance modulatory and efflux-inhibitory activities of capsaicinoids and capsinoids. Bioorganic Chem. 2019, 82, 378-384. [CrossRef]

30. Machado, D.; Couto, I.; Perdigão, J.; Rodrigues, L.; Portugal, I.; Baptista, P.; Veigas, B.; Amaral, L.; Viveiros, M. Contribution of efflux to the emergence of isoniazid and multidrug resistance in Mycobacterium tuberculosis. PLoS ONE 2012, 7, e34538. [CrossRef] [PubMed]

31. Christensen, G.D.; Simpson, W.A.; Younger, J.J.; Baddour, L.M.; Barrett, F.F.; Melton, D.M.; Beachey, E.H. Adherence of coagulasenegative staphylococci to plastic tissue culture plates: A quantitative model for the adherence of staphylococci to medical devices. J. Clin. Microbiol. 1985, 22, 996-1006. [CrossRef]

32. O'Toole, G.A. Microtiter dish Biofilm formation assay. J. Vis. Exp. 2011. [CrossRef]

33. Abidi, S.H.; Ahmed, K.; Sherwani, S.K.; Bibi, N.; Kazmi, U.S. Detection of Mycobacterium Smegmatis Biofilm and its Control by Natural Agents. Int. J. Curr. Microbiol. Appl. Sci. 2014, 3, 801-812.

34. Kwasny, S.M.; Opperman, T.J. Static biofilm cultures of Gram-positive pathogens grown in a microtiter format used for anti-biofilm drug discovery. Curr. Protoc. Pharmacol. 2010, 50, 13A.8. [CrossRef] [PubMed] 


\section{Short Biography of Authors}

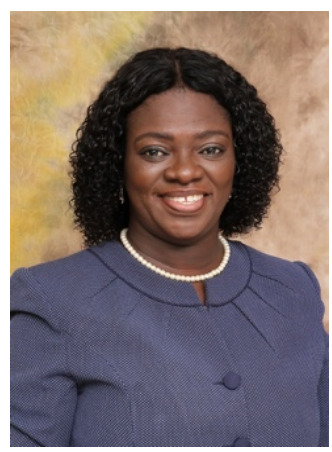

Dr. (Mrs.) Cynthia Amaning Danquah is a Senior Lecturer at the Department of Pharmacology, Kwame Nkrumah University of Science and Technology (KNUST), Kumasi, Ghana. She pursued an interdisciplinary PhD in Drug Discovery to tackle antimicrobial resistance at the School of Pharmacy, University College London, UK and Birkbeck, University of London, UK. Her MPhil (Pharmacology) and Bachelor of Pharmacy (Hons) were obtained from KNUST. Natural product drug discovery, tuberculosis, antimicrobial resistance and natural product pharmacology and toxicology are her research areas of interest. She is a Pharmacist with passion for infectious diseases of public health concern and a member of a range of professional bodies including the British Pharmacological Society (BPS), the British Society for Antimicrobial Chemotherapy (BSAC), International Society for Pharmacovigilance (ISOP), the West African Network of Natural Products Research Scientists (WANPRESS), International Pharmaceutical Federation (FIP), Pharmaceutical Society of Ghana (PSGH), an affiliate of the African Academy of Science (AAS) and the Country ambassador for the American Society for Microbiology (ASM).

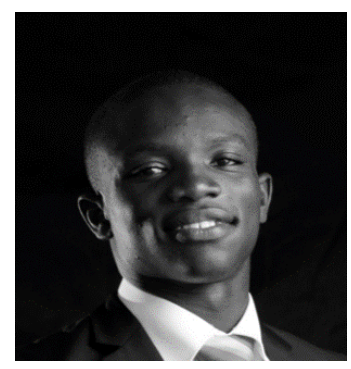

Michael Tetteh is a research assistant at the Department of Pharmacology, Kwame Nkrumah University of Science and Technology (KNUST). He graduated with a BSc. (Hon) in Herbal Medicine at KNUST in 2016. He worked as a teaching and research assistant in the Department of Pharmacology KNUST, a medical intern at Tetteh Quarshie Memorial Hospital and a research intern at the Center for Plant Medicine Research all in Ghana. Natural product drug discovery against resistant infectious disease is his research area of interest. He is a member of the British Society for Antimicrobial Chemotherapy, the American Society for Microbiology and the Ghana Association of Medical Herbalist.

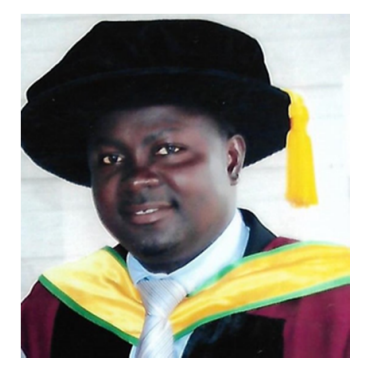

Dr. Isaac Kingsley Amponsah is a Senior lecturer in Pharmacognosy at the Kwame Nkrumah University of Science and Technology (KNUST). He holds a Doctor of philosophy in Pharmacognosy (completed 2012) and a Bachelor of Pharmacy degree (completed 2005), all from KNUST. He has a certificate in Good manufacturing practices (GMP) and good agricultural collection practices (GCAP) from Hanoi University of Pharmacy in Vietnam and i+solutions, Amsterdam, Netherlands. His research focuses on phytochemical investigation of biologically active plant medicines and the quality control of herbal products using various analytical methods. His current research includes plant constituents as biofilm inhibitors for the mitigation of microbial resistance, plant constituents and formulations against Mycobacterium ulcerans (implicated in Buruli ulcer) and standardisation of herbal products. Dr Isaac Kingsley Amponsah is a member of the society for medicinal plants and natural product research. He serves as an ECOWAS expert committee member on traditional medicine. He is also a member of the governing board of the traditional medicine practice council of Ghana and Chairman of its education committee.

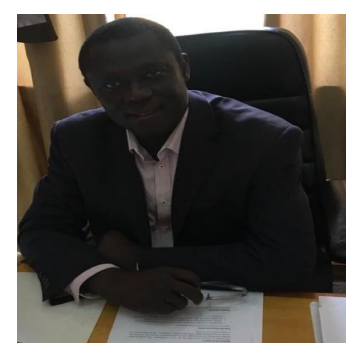

Professor Kwame Ohene Buabeng is a Clinical Pharmacologist and Associate Professor of Pharmacy Practice at Kwame Nkrumah University of Science and Technology (KNUST), Kumasi, Ghana. He is the Vice President of the Ghana College of Pharmacists (GCPharm) and Chair of the Division of Pharmaceutical Care, GCPharm. He is also Deputy Chair of the multi-sectoral, multi-stakeholder coordinating platform on Antimicrobial Resistance in Ghana, and a Member of the National Medicines Selection Committee for the Development of Standard Treatment Guidelines (STG) and Essential Medicines List (EML). Prof Ohene Buabeng has B. Pharm degree, MSc in Clinical Pharmacology and PhD in Pharmacy (Social Pharmacy-Health Systems and Drug Utilization Research) as well as Certificate in Public Health Science, Research and Ethics. His research interest is in prevention, pharmacotherapy and control of Infectious Diseases and Cardio-metabolic disorders. 

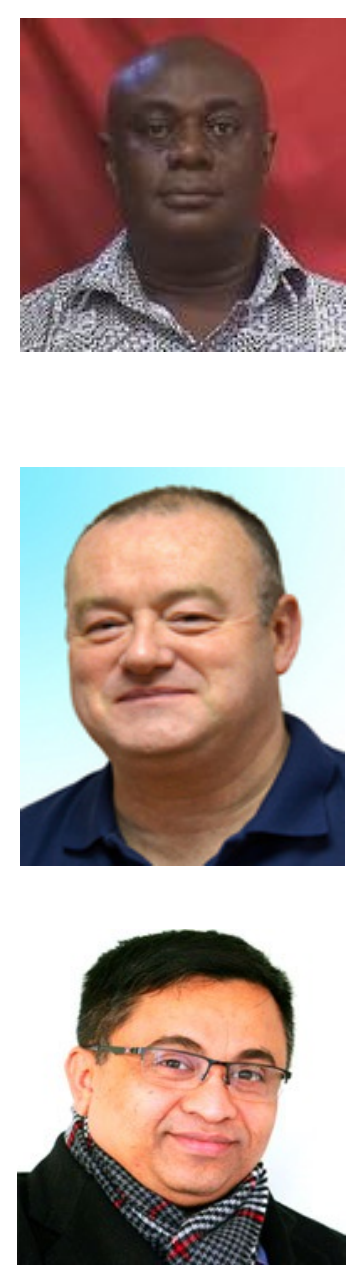

Professor Abraham Yeboah Mensah obtained his PhD at King's College, London, United Kingdom in1999 and his Bachelor of Pharmacy (Hons) in 1990 from KNUST. He is a Pharmacist and a lecturer in Pharmacognosy. His research interest is in natural products with biological activities. Prof Yeboah Mensah is a Commonwealth Scholar and Fellow and a recipient of the prestigious Welcome Trust (United Kingdom) Research Development Award in Tropical Medicine (2002-2004). He is also a scholar of the University of Michigan African Presidential Scholar Program. Other awards received by Prof Abraham Yeboah Mensah include the German Academic Exchange Service Award (DAAD). He collaborates with a number of scientists from several countries including Germany, United States and the United Kingdom. He is a Fellow of the Pharmaceutical Society of Ghana (PSGH) and a Fellow of the Ghana College of Pharmacist.

Professor Simon Gibbons is a Professor of Natural Product Chemistry at the University of East Anglia School of Pharmacy, UK. He is founding Editor-in-Chief of Phytochemistry Letters and Co-editor of Progress in the Chemistry of Organic Natural Products ("Zechmeister"). Simon was past President of the Phytochemical Society of Europe and has served on the UK Government's Advisory Council on the Misuse of Drugs (ACMD). He currently is a member of the Home Office's Cannabis Based Medicinal Products Committee. He has special interests in medicinal natural products, phytochemistry and antimicrobial natural products from plants and microbes. His group specialises in the isolation and structure elucidation of natural products that are active against drugresistant bacteria. He also is interested in compounds that modify bacterial resistance. These may be plasmid transfer inhibitors or efflux inhibitors.

Professor Sanjib Bhakta is a Professor of Molecular Microbiology and Biochemistry, Department of Biological Sciences, Institute of Structural and Molecular Biology (ISMB), Birkbeck, University of London, UK. He is the Programme Director of MRes Global Infectious Diseases, Department of Biological Sciences, Assistant Dean (Strategic) Internationalisation and Partnership, School of Science, Birkbeck. Professor Bhakta's research group at the ISMB-Mycobacteria Research Laboratory works on global infectious diseases such as tuberculosis (TB) and international health challenges with antibiotic resistance. His main research interests are focused around characterising the physiology of the different metabolic states of Mycobacterium spp., tackling antimicrobial resistance through validating novel therapeutic targets, from identifying hits to optimising novel leads and repurposing existing immunomodulatory drugs to cure TB. 